\title{
SOME PROPERTIES OF ORTHOGONAL POLYNOMIALS SATISFYING FOURTH ORDER DIFFERENTIAL EQUATIONS
}

\author{
by R. G. CAMPOS and L. A. AVILA
}

(Received 29 June, 1993)

1. Introduction. In the last few years, there has been considerable interest in the properties of orthogonal polynomials satisfying differential equations (DE) of order greater than two, their connection to singular boundary value problems, their generalizations, and their classification as solutions of second order DE (see for instance [2-8]). In this last interesting problem, some known facts about the classical orthogonal polynomials can be incorporated to connect these two sets of families and yield some nontrivial results in a very simple way. In this paper we only work with the nonclassical Jacobi type, Laguerre type and Legendre type polynomials, and we show how they can be connected with the classical Jacobi, Laguerre and Legendre polynomials, respectively; at the same time we obtain certain bounds for the zeros of the first ones by using a system of nonlinear equations satisfied by the zeros of any polynomial solution of a second order differential equation which, for the classical polynomials is known since Stieltjes and concerns the electrostatic interpretation of the zeros $[10$, Section $6.7 ; 9,1]$. We also correct an expression for the second order differential equation of the Legendre type polynomials that circulates through the literature.

2. Differential equations and zeros of polynomial solutions. In this section we review some known and basic facts concerning the zeros of polynomial solutions of linear operators.

For the sake of generality let us take a linear DE of $n$th order.

Proposition 1. Let $f_{n}(x)=\prod_{k=1}^{n}\left(x-x_{k}\right)$ be a polynomial of degree $n$ with distinct (complex) zeros $x_{1}, x_{2}, \ldots, x_{n}$, satisfying the linear differential equation

$$
\sum_{k=0}^{n} a_{k}(x) \frac{d^{k}}{d x^{k}} f_{n}(x)=0 .
$$

Then the zeros $x_{j}, j=1,2, \ldots, n$, satisfy the following system of nonlinear equations

$$
\begin{aligned}
n a_{n}\left(x_{j}\right)\left[\sum_{i_{1} \neq i_{2} \neq \ldots \neq i_{n-1}}^{n} \frac{1}{\left(x_{j}-x_{i_{1}}\right) \ldots\left(x_{j}-x_{i_{n-1}}\right)}\right] & \\
& +(n-1) a_{n-1}\left(x_{j}\right)\left[\sum_{i_{1} \neq i_{2} \neq \ldots \neq i_{n-2}}^{n} \frac{1}{\left(x_{j}-x_{i_{1}}\right) \ldots\left(x_{j}-x_{i_{n-2}}\right)}\right] \\
& \quad \ldots+2 a_{2}\left(x_{j}\right)\left[\sum_{i}^{n} \frac{1}{\left(x_{j}-x_{i}\right)}\right]+a_{1}\left(x_{j}\right)=0,
\end{aligned}
$$

$j=1,2, \ldots, n$. The symbol $\sum^{\prime}$ means the sum over $i \neq j$.

REMARK. If the order of the differential equation (1) is 2 , we have the important 
case of the classical orthogonal polynomials and their zeros. All of these zeros satisfy an equation of the form

$$
\sum_{i=1}^{n} \frac{1}{\left(x_{j}-x_{i}\right)}+\frac{\gamma^{\prime}\left(x_{j}\right)}{\gamma\left(x_{j}\right)}=0, \quad j=1,2, \ldots, n,
$$

where $\gamma(x)$ is a function related to the weight function of each family of polynomials; see for instance [1]. The explicit form of this equation for the Jacobi case was known already by Stieltjes; all the three cases have an electrostatic interpretation and are involved in a problem of maximization $[\mathbf{1 0}$, Section $6.7 ; 9]$.

Proof. This is a very easy matter and follows Laguerre [10, pp 117-120]. Define the polynomial of degree $n-1 g(x)=\prod_{k \neq j}^{n}\left(x-x_{k}\right)$. Thus, $f(x)=\left(x-x_{j}\right) g(x)$, and therefore

$$
f^{(m)}\left(x_{j}\right)=m g^{(m-1)}\left(x_{j}\right), \quad m=1,2, \ldots
$$

Now, evaluate (1) at $x_{j}$ and divide by $f^{\prime}\left(x_{j}\right) \neq 0$ to yield

$$
\sum_{k=1}^{n} a_{k}\left(x_{j}\right) \frac{f^{(k)}\left(x_{j}\right)}{f^{\prime}\left(x_{j}\right)}=0
$$

and then use (4) to obtain

$$
\sum_{k=1}^{n} k a_{k}\left(x_{j}\right) \frac{g^{(k-1)}\left(x_{j}\right)}{g\left(x_{j}\right)}=0 .
$$

Remember that $g(x)$ is a polynomial of degree $n-1$. The $(k-1)$ th derivative removes from $g(x)(k-1)$ factors of the form $\left(x-x_{i_{1}}\right)\left(x-x_{i_{2}}\right) \ldots\left(x-x_{i_{k-1}}\right)$, all of them different, and produces a sum over these indexes; thus, we have that

$$
\frac{g^{(k-1)}\left(x_{j}\right)}{g\left(x_{j}\right)}=\sum_{i_{1} \neq i_{2} \neq \ldots \neq i_{k-1}}^{n} \frac{1}{\left(x_{j}-x_{i_{1}}\right) \ldots\left(x_{j}-x_{i_{k-1}}\right)}
$$

and therefore (2) holds.

Equation (3) plays an important role in obtaining the main results of this paper, so we should spend a little more time on it.

As remarked above, the zeros of the classical orthogonal polynomials satisfy (3) where

$$
\gamma(x)=\left\{\begin{array}{cl}
\gamma\}^{(r s)}(x) \equiv(1-x)^{(r+1) / 2}(1+x)^{(s+1) / 2} & \text { for Jacobi } \\
\gamma_{L}^{\alpha}(x) \equiv x^{(\alpha+1) / 2} e^{-x / 2} & \text { for Laguerre } \\
\gamma_{H}(x) \equiv e^{-x^{2 / 2}} & \text { for Hermite }
\end{array}\right.
$$

Note that $\gamma(x)$ vanishes at the extremes of the intervals of orthogonality and $\ln (\gamma(x))$ is a concave function in each case. A theorem proved by Popoviciu [9] states sufficient conditions on a function $\gamma(x)$ to give the unique solution of (3):

(i) $\gamma(x)$ should be in $C^{1}(a, b), a, b$ finite,

(ii) $\gamma(a)=\gamma(b)=0$,

(iii) $\ln \gamma(x)$ should be a concave function in $[a, b]$. 
Such a solution consists in a set of (distinct) points contained entirely in $(a, b)$. The part of the proof corresponding to the existence makes use of the continuous and positive function of $n$ variables $a \leq z_{j} \leq b, j=1,2, \ldots, n$,

$$
T(z)=\prod_{l=1}^{n} \gamma\left(z_{l}\right) \prod_{i>j=1}^{n}\left(z_{i}-z_{j}\right)
$$

that vanishes whenever $z_{j}=a, b$ or $z_{k}=z_{j}$, attaining a maximum inside that region. (3) is the condition for extremum.

3. Bounds and relationships. It is known [3-8] that the orthogonal polynomials satisfying a fourth order DE can also be obtained as solutions of a second order DE of the form

$$
a_{2}(x, n) f_{n}^{\prime \prime}(x)+a_{1}(x, n) f_{n}^{\prime}(x)+a_{0}(x, n) f_{n}(x)=0
$$

where the coefficients depend not only on $x$, but also on $n$. However, as polynomial solutions of a second order DE, their zeros satisfy (2) with $a_{j}(x) \equiv 0$ for $j>2$. The resulting equation plays the main role in the following.

For the sake of simplicity we separate each case and summarize the features we need of the corresponding polynomial. The notation is that of [4].

Jacobi type polynomials. They are denoted by $S_{n}(x), n=0,1, \ldots$ and can be obtained through

$$
S_{n}(x)=\frac{n ! \sum_{k=0}^{n}(-1)^{n-k}\left[\begin{array}{l}
n \\
k
\end{array}\right](1+\alpha)_{n+k}[k(n+\alpha)(n+1)+(k+1) M] x^{k}}{(k+1) !(1+\alpha)_{n}},
$$

where the Pochhammer symbol $(a)_{n}$ is defined by $(a)_{n}=a(a+1)(a+2) \ldots(a+n-1)$. (The factor $n$ ! is missing in [4].) These polynomials are solutions of the Sturm-Liouville $\mathrm{DE}$

$$
\begin{aligned}
& \left(\left[(1-x)^{\alpha+4}-2(1-x)^{\alpha+3}+(1-x)^{\alpha+2}\right] S_{n}^{\prime \prime}\right)^{\prime \prime} \\
& \quad+\left(\left[(2 \alpha+2+M)(1-x)^{\alpha+2}-2(\alpha+2+M)(1-x)^{\alpha+1}\right] S_{n}^{\prime}\right)^{\prime}=\lambda_{n}^{J}(1-x)^{\alpha} S_{n}
\end{aligned}
$$

where

$$
\begin{aligned}
\lambda_{n}^{J}= & 2(\alpha+2)(\alpha+1+M) n+\left(\alpha^{2}+9 \alpha+14+2 M\right) n(n-1) \\
& +2(\alpha+4) n(n-1)(n-2)+n(n-1)(n-2)(n-3)
\end{aligned}
$$

and their squared norm is

$$
\left\|S_{n}\right\|^{2}=\int_{0}^{1} S_{n}^{2}(x)(1-x)^{\alpha} d x+\frac{1}{M} S_{n}^{2}(0)=\frac{[M+n(n+\alpha)][M+(n+1)(n+\alpha+1)]}{(2 n+\alpha+1)} .
$$

In this case we have an equation like (6) where

$$
\begin{gathered}
a_{2}(x, n)=x\left[A_{J} x^{2}-\left(A_{J}+M\right) x+M\right] \\
a_{1}(x, n)=\left[2 M\left(M^{2}+\alpha+1+\lambda_{n}^{J}\right)+\alpha A_{J}\right] x^{2}-\left(A_{J}+M \alpha+3 M\right) x+2 M \\
a_{0}(x, n)=\left[(-22 \alpha+2 M+110) \lambda_{n}^{J}-\eta_{n}^{J}\right] x+\lambda_{n}^{J}
\end{gathered}
$$


and

$$
\begin{aligned}
A_{J}=M^{2}+M \alpha+M+\lambda_{n}^{J}=(2 n+\alpha+1)\left\|S_{n}\right\|^{2}, \\
\eta_{n}^{J}=-\left(42 \alpha^{3}+342 \alpha^{2}+732 \alpha+432+6 M^{2}+438 M+309 M \alpha+3 M^{2} \alpha+45 M \alpha^{2}\right) n \\
-\left(18 \alpha^{3}+270 \alpha^{2}+1188 \alpha+1440+3 M^{2}+219 M+45 M \alpha\right) n(n-1) \\
+\left(\alpha^{3}-21 \alpha^{2}-274 \alpha-696\right) n(n-1)(n-2)+\left(3 \alpha^{2}+15 \alpha-12\right) n(n-1)(n-2)(n-3) \\
+(18+3 \alpha) n(n-1)(n-2)(n-3)(n-4)+n(n-1)(n-2)(n-3)(n-4)(n-5) .
\end{aligned}
$$

For this case, (2) can be cast in the form (3) with $\gamma(x)$ given by

$$
\Gamma_{J}(x)=\left(A_{J} x-M\right)^{\mu / \rho} x(1-x)^{v / \rho}
$$

where

$$
\begin{aligned}
\mu= & (8 \alpha-3) M^{4}+\left(8 \alpha^{2}-2 \alpha-3\right) M^{3}+\left(-3 \alpha^{2}+8 \alpha \lambda_{n}^{J}-4 \alpha-2 \lambda_{n}^{J}-2\right) M^{2} \\
& -\lambda_{n}^{J}(4 \alpha+5) M-\left(\lambda_{n}^{J}\right)^{2}, \\
v= & 2 M^{5}+(\alpha+1) M^{4}+\left(-2 \alpha+2 \lambda_{n}^{J}+1\right) M^{3}+\left(\alpha^{3}+\alpha^{2}+2 \alpha+2\right) M^{2} \\
& +\lambda_{n}^{J}\left(2 \alpha^{2}+2 \alpha+3\right) M+(\alpha+1)\left(\lambda_{n}^{J}\right)^{2}, \\
\rho= & 2\left[M^{4}+(2 \alpha+1) M^{3}+\left(\alpha^{2}+\alpha+2 \lambda_{n}^{J}\right) M^{2}+\lambda_{n}^{J}(2 \alpha+1) M+\left(\lambda_{n}^{J}\right)^{2}\right] .
\end{aligned}
$$

Due to the relation between this function and $\gamma_{J}^{(r, s)}(2 x-1)$ (see (5)), it is convenient to work out these indices a bit more. As polynomials in $M$, they can be rewritten as

$$
\begin{gathered}
\begin{aligned}
\mu= & (8 \alpha-3) M^{4}+\left[8(2 n+1) \alpha^{2}+2\left(8 n^{2}+6 n-1\right) \alpha-\left(4 n^{2}+4 n+3\right)\right] M^{3} \\
+ & {\left[8 n(n+1) \alpha^{3}+\left(16 n^{3}+22 n^{2}-2 n-3\right) \alpha^{2}+2\left(4 n^{4}+6 n^{3}-3 n^{2}-10 n-2\right) \alpha\right.} \\
- & \left.2\left(n^{4}+2 n^{3}+6 n^{2}+5 n+1\right)\right] M^{2}-n(n+\alpha+1)[(n+1)(n+\alpha)(4 \alpha+5)+2] M \\
- & n(n+1)(n+\alpha)(n+\alpha+1), \\
v= & {[M+n(n+\alpha)][M+(n+1)(n+\alpha+1)]\left\{2 M^{3}-(\alpha+1) M^{2}\right.} \\
& +\left[(2 n+1) \alpha^{2}+2 n(n+2) \alpha+2\left(n^{2}+n+1\right)\right] M \\
& +n(n+1)(n+\alpha)(n+\alpha+1)(\alpha+1)\}
\end{aligned} \\
\rho=2[M+n(n+\alpha)][M+(n+1)(n+\alpha+1)][M+n(n+\alpha+1)][M+(n+1)(n+\alpha)] .
\end{gathered}
$$

A simple analysis shows that $\mu$ and $\rho$ are relatively prime for all $n$ and $\alpha>-1$. Some numerical trials reveal the existence of curves within some regions of the $M-\alpha$ plane ( $n$ fixed) on which $\mu=0$. For example, if $\alpha>3 / 8$ and $n$ is fixed, four real values of $M$ are obtained for which $\mu=0$. For such curves, the functions $\Gamma_{J}(x)$ and $\gamma_{j}^{(r)}(2 x-1)$ (with a proper selection of the parameters of the latter) generate the same polynomials through (3). This result can also be obtained by considering the values for which the factor $\left(A_{J} x-M\right)$ becomes a constant different from zero (whether positive or negative is immaterial because of the logarithmic derivative in (3)), i.e., for $A_{J} \equiv[M+n(n+\alpha)]$ $[M+(n+1)(n+\alpha+1)]=0$; but some caution is necessary because the power of 
such a factor diverges at these points and, on the other hand, $S_{n}(x)$ collapses to a polynomial of degree $n-1$ if $M=-n(n+\alpha)$.

Let $M>0$ and $\alpha>-1$. Thus

$$
\xi=\frac{M}{A_{J}}=\frac{M}{(2 n+\alpha+1)\left\|S_{n}\right\|^{2}}
$$

is a positive number less than 1 that approaches zero as one of the parameters $M, \alpha$ or $n$ is increased. In this region of the $M-\alpha$ plane, $\rho$ is positive. If we exclude the points at which $\mu=0$, we have that the function $\Gamma_{J}(x)$ vanishes or diverges at $x=\xi$, as $\mu$ is positive or negative, and the continuity of the zeros on its parameters (referred to $\mu=0$ ) guarantees that they must be contained in $(\xi, 1)$. On the other hand, if $\xi \rightarrow 0, \Gamma_{J}(x)$ approaches (except for a constant)

$$
\gamma_{j}^{((2 v / \rho)-1,(2 \mu / \rho)+1)}(2 x-1)=x^{(\mu / \rho)+1}(1-x)^{v / \rho} .
$$

Thus, we have proved the following result.

Proposition 2. Let $S_{n}(x)$ and $P_{n}^{(r, s)}(x)$ be the Jacobi type and classical Jacobi polynomials, respectively.

(i) If $M>0$ and $\alpha>-1$,

$$
\xi=\frac{M}{[M+n(n+\alpha)][M+(n+1)(n+\alpha+1)]}
$$

is a lower bound for the zeros of $S_{n}(x)$.

(ii) If $\mu=0$,

$$
S_{n}(x)=\left[\frac{(n+\alpha+1)_{n}[M+n(n+\alpha)]}{\left(\begin{array}{c}
2 n+2 v / \rho \\
n
\end{array}\right)}\right] P_{n}^{((2 v / \rho)-1,1)}(2 x-1) .
$$

(iii) Furthermore, if $\xi \rightarrow 0$,

$$
S_{n}(x) \rightarrow\left[\frac{(n+\alpha+1)_{n}[M+n(n+\alpha)]}{\left(\begin{array}{c}
2 n+2(\mu+v) / \rho \\
n
\end{array}\right)}\right] P_{n}^{((2 v / \rho)-1,(2 \mu / \rho)+1)}(2 x-1) .
$$

In these formulas we have taken into account the proper standardization of each polynomial.

Laguerre type polynomials. These polynomials are denoted by $R_{n}(x), n=$ $0,1, \ldots$ and have the form

$$
R_{n}(x)=\sum_{k=0}^{n} \frac{(-1)^{k}}{(k+1) !} \frac{n !}{(n-k) ! k !}[k(R+n+1)+R] x^{k} .
$$

They satisfy the Sturm-Liouville DE

$$
\left[x^{2} e^{-x} R_{n}^{\prime \prime}(x)\right]^{\prime \prime}-\left\{[2(R+1) x+2] e^{-x} R_{n}^{\prime}(x)\right\}^{\prime}=\lambda_{n}^{L} e^{-x} R_{n}(x),
$$


where $R$ is a real parameter different from zero and $\lambda_{n}^{L}=2(R+1) n+n(n-1)$. Their norm is given by

$$
\left\|R_{n}\right\|^{2}=\int_{0}^{\infty} R_{n}^{2}(x) e^{-x} d x+\frac{1}{R} R_{n}^{2}(0)=(R+n)(R+n+1) .
$$

These polynomials are also solutions of an equation like (6) where

$$
\begin{gathered}
a_{2}(x, n)=\left(R^{2}+R+\lambda_{n}^{L}\right) x^{2}-R x \\
a_{1}(x, n)=-\left(R^{2}+R+\lambda_{n}^{L}\right) x^{2}+\left(R^{2}+2 R+\lambda_{n}^{L}\right) x-2 R \\
a_{0}(x, n)=\left(2 R \lambda_{n}^{L}+22 \lambda_{n}^{L}-\eta_{n}^{L}\right) x-\lambda_{n}^{L}
\end{gathered}
$$

and

$$
\eta_{n}^{L}=\left(3 R^{2}+45 R+42\right) n+n(n-1)(10-n) .
$$

In this case, (2) can be written in the form (3) with $\gamma(x)$ given by

$$
\Gamma_{L}(x)=\left(A_{L} x-R\right)^{-1 / 2} x e^{-x / 2},
$$

where $A_{L}$ turns out to be the squared norm of $R_{n}$ :

$$
A_{L}=R^{2}+R+\lambda_{n}^{L}=\left\|R_{n}\right\|^{2} .
$$

$\Gamma_{L}(x)$ vanishes at the extremes of the interval $[0, \infty)$, but diverges at

$$
\xi=\frac{R}{(R+n)(R+n+1)}
$$

whenever $(R+n)(R+n+1) \neq 0$. If $R>0, \xi$ is an interior point on the interval and $\Gamma_{L}(x)$ takes imaginary values for $0<x<\xi$. Therefore, $\xi$ must be a lower bound for the real solutions of (3), and in particular for the zeros of $R_{n}(x)$. Besides this, if we let $\xi \rightarrow 0$, $\Gamma_{L}(x)$ approaches

$$
\gamma_{L}^{0}(x)=x^{1 / 2} e^{-x / 2},
$$

except for a constant, and according to (5) this $\gamma(x)$ generates the zeros of Laguerre polynomials $L_{n}^{0}(x)$. Similarly, if $R=-n-1$, or $R=-n, \Gamma_{L}(x)$ is proportional to $\gamma_{L}^{1}(x)$, but now (5) generates the zeros of $L_{n}^{1}(x)$ for the first choice of $R$ and of $L_{n-1}^{1}(x)$ for the latter (note that $R_{n}(x)$ collapses to a polynomial of degree $n-1$ if $R=-n$ ). Thus, we have proved the following

PROPOSIrION 3. Let $R_{n}(x)$ and $L_{n}^{\alpha}(x)$ be the Laguerre type and classical Laguerre polynomials, respectively.

(i) If $R>0$,

$$
\xi=\frac{R}{(R+n)(R+n+1)}
$$

is a lower bound for the zeros of $R_{n}(x)$.

(ii) If $\xi \rightarrow 0$, then $R_{n}(x) \rightarrow(R+n) L_{n}^{0}(x)$.

(iii) If $R=-n-1$, or $R=-n$, then $R_{n}(x)=-L_{n}^{1}(x)$ or $R_{n}(x)=-L_{n-1}^{1}(x)$ respectively. 
In these formulas we have taken into account the proper standardization of each polynomial.

Legendre type polynomials. These polynomials denoted by $P_{n}^{\alpha}(x), n=$ $0,1,2, \ldots$, have the form

$$
P_{n}^{\alpha}(x)=\sum_{k=0}^{[n / 2]} \frac{(-1)^{k}(2 n-2 k) !\left[\alpha+\frac{1}{2} n(n-1)+2 k\right]}{2^{n} k !(n-k) !(n-2 k) !} x^{n-2 k},
$$

where $[v]$ denotes the greatest integer $\leq v$. This family satisfies the Sturm-Liouville DE

where

$$
\left[\left(x^{2}-1\right)^{2} P_{n}^{\alpha \prime \prime}(x)\right]^{\prime \prime}+4\left\{\left[\alpha\left(x^{2}-1\right)-2\right] P_{n}^{\alpha \prime}(x)\right\}^{\prime}=\lambda_{n}^{P} P_{n}^{\alpha}(x),
$$

$$
\lambda_{n}^{P}=n(n+1)\left(n^{2}+n+4 \alpha-2\right) .
$$

In this case the norm is

$$
\begin{aligned}
\left\|P_{n}^{\alpha}\right\|^{2} & =\int_{-1}^{1}\left(P_{n}^{\alpha}(x)\right)^{2} \frac{\alpha}{2} d x+\frac{1}{2}\left(P_{n}^{\alpha}(-1)\right)^{2}+\frac{1}{2}\left(P_{n}^{\alpha}(1)\right)^{2} \\
& =\alpha\left[\alpha+\frac{(n-1) n}{2}\right]\left[\alpha+\frac{(n+1)(n+2)}{2}\right] /(2 n+1) .
\end{aligned}
$$

For these polynomials we have an equation like (6) where

$$
\begin{gathered}
a_{2}(x, n)=\left(x^{2}-1\right)\left[\left(4 \alpha^{2}+4 \alpha+\lambda_{n}^{P}\right) x^{2}-\left(4 \alpha^{2}-4 \alpha+\lambda_{n}^{P}\right)\right] \\
a_{1}(x, n)=2 x\left[\left(4 \alpha^{2}+4 \alpha+\lambda_{n}^{P}\right) x^{2}-\left(4 \alpha^{2}-12 \alpha+\lambda_{n}^{P}\right)\right] \\
a_{0}(x, n)=-\eta_{n}^{P} x^{2}+\left(\eta_{n}^{P}-4 \lambda_{n}^{P}\right)
\end{gathered}
$$

and

$$
\eta_{n}^{P}=n(n+1)\left[4 \alpha^{2}+4\left(n^{2}+n+1\right) \alpha+(n-1) n(n+1)(n+2)\right] .
$$

(We have found in the literature an incorrect expression for the $a_{0}(x, n)$ term in such a differential equation.)

We have now that (2) can be written in the form (3) with $\gamma(x)$ given by

$$
\Gamma_{P}(x)=\left(A-B x^{2}\right)^{-1 / 2}\left(1-x^{2}\right),
$$

where $A$ and $B$ come out to be related to the norm square of $P_{n}^{\alpha}(x)$ :

$$
\begin{gathered}
A=[2 \alpha+n(n+1)][2 \alpha+(n-1)(n+2)]=\frac{4(2 n+1)}{\alpha}\left\|P_{n}^{\alpha}\right\|^{2}-8 \alpha, \\
B=[2 \alpha+n(n-1)][2 \alpha+(n+1)(n+2)]=\frac{4(2 n+1)}{\alpha}\left\|P_{n}^{\alpha}\right\|^{2} .
\end{gathered}
$$

$\Gamma_{P}(x)$ vanishes at the extremes of the interval $[-1,1]$, and diverges at

$$
\xi= \pm \sqrt{\frac{A}{B}}= \pm \sqrt{1-\frac{2 \alpha^{2}}{(2 n+1)\left\|P_{n}^{\alpha}\right\|^{2}}}
$$

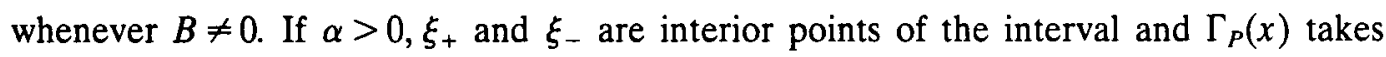


imaginary values for $0<\xi_{+}<|x|$. Therefore, $\xi_{+}\left(\xi_{-}\right)$must be an upper (lower) bound for the real solutions of (3), and in particular for the zeros of $P_{n}^{\alpha}(x)$. Besides this, if we let $\xi_{+} \rightarrow 1, \Gamma_{P}(x)$ approaches

$$
\gamma\}^{(0,0)}(x)=\left(1-x^{2}\right)^{1 / 2}
$$

except for a constant, and according to (5), this $\gamma(x)$ generates the zeros of Jacobi (Legendre) polynomials $P_{n}^{(0,0)}(x)$. Similarly, if $\alpha=-(n+1)(n+2) / 2$ or $\alpha=-n(n-1) / 2$, $\Gamma_{P}(x)$ is proportional to $\gamma_{j}^{(1,1)}(x)$, and now (5) generates the zeros of $P_{n}^{(1,1)}(x)$ for the first choice of $\alpha$ and of $P_{n-2}^{(1,1)}(x)$ for the latter $\left(P_{n}^{\alpha}(x)\right.$ collapses to a polynomial of degree $n-2$ if $\alpha=-n(n-1) / 2)$. Thus, we have proved the following

Proposition 4. Let $P_{n}^{\alpha}(x), P_{n}(x)$ and $P_{n}^{(\alpha, \beta)}(x)$ be the Legendre type, classical Legendre and classical Jacobi polynomials, respectively. Here, $P_{n}(x) \equiv P_{n}^{(0,0)}(x)$.

(i) If $\alpha>0$, the zeros of $P_{n}^{\alpha}(x)$ are contained in $(-\xi, \xi)$, where

$$
\xi=\sqrt{\frac{[2 \alpha+n(n+1)][2 \alpha+(n-1)(n+2)]}{(2 \alpha+n(n-1)][2 \alpha+(n+1)(n+2)]}}
$$

(ii) If $\xi \rightarrow 1$, then $P_{n}^{\alpha}(x) \rightarrow[\alpha+n(n-1) / 2] P_{n}(x)$.

(iii) If $\alpha=-(n+1)(n+2) / 2$ or $\alpha=-n(n-1) / 2$, then $P_{n}^{\alpha}(x)=-[(n+2) / 2] P_{n}^{(1,1)}(x)$, or $P_{n}^{\alpha}(x)=-(n / 2) P_{n-2}^{(1,1)}(x)$, respectively.

Again, in these formulas we have taken into account the proper standardization of each polynomial.

To conclude this paper, we remark that some of the preceding results can also be obtained by a simple and direct comparison of both DE. This is true for some cases but not for all of them. Most of the limit cases are not so obvious, and the obtainment of the bounds can not be worked out in such a way.

4. ACKNOWLEDGMENTS. The authors are grateful for the partial support of the Dirección General de Investigación Científica y Superación Académica (DGICSA), SEP.

\section{REFERENCES}

1. R. G. Campos, Some Properties of the Zeros of Polynomial Solutions of Sturm-Liouville Equations, SIAM J. Math. Anal. 18 (1987), 1664-1668.

2. J. S. Dehesa, E. Buendía and M. A. Sánchez-Buendía, On the polynomial solutions of ordinary differential equations of the fourth order, J. Math. Phys. 26 (1985), 1547-1552.

3. T. H. Koornwinder, Orthogonal polynomials with weight function $(1-x)^{\alpha}(1+x)^{\beta}+$ $M \delta(x+1)+N \delta(x-1)$, Canad. Math. Bull. $27(1984), 205-214$.

4. A. M. Krall, A review of orthogonal polynomials satisfying boundary value problems, in "Orthogonal Polynomial and Their Applications", (M. Alfaro et al., eds.), Lect. Notes in Math., 1329, Springer-Verlag, Berlin (1988), 73-97.

5. L. L. Littlejohn, The Krall polynomials as solutions to a second order differential equation, Canad. Math. Bull. 26 (1983), 410-417.

6. L. L. Littlejohn, Orthogonal polynomial solutions to ordinary and partial differential equations, In "Orthogonal Polynomial and Their Applications", (M. Alfaro et al., eds.), Lect. Notes in Math., 1329, Springer-Verlag, Berlin (1988), 98-124.

7. L. L. Littlejohn and A. M. Krall, Orthogonal polynomials and higher order singular Sturm-Liouville systems, Acta Appl. Math. 17 (1989), 99-170. 
8. L. L. Littlejohn and A. M. Shore, Nonclassical orthogonal polynomials as solutions to second order differential equations, Canad. Math. Bull. 25 (1982), 291-295.

9. T. Popoviciu, Sur certains problèmes de maximum de Stieltjes, Bull. Math. Soc. Roumaine Sci., 38 (1936), 73-96.

10. G. Szegö, Orthogonal polynomials (American Mathematical Society, Providence, RI, fourth edition, 1976).

Escuela de Física y Matemáticas

Universidad MichoaCana, 58060 Morelia

Michoacán, México 